\title{
Peroxiredoxins and the Regulation of Cell Death
}

\author{
Mark B. Hampton*, and Karina M. O’Connor
}

\begin{abstract}
Cell death pathways such as apoptosis can be activated in response to oxidative stress, enabling the disposal of damaged cells. In contrast, controlled intracellular redox events are proposed to be a significant event during apoptosis signaling, regardless of the initiating stimulus. In this scenario oxidants act as second messengers, mediating the post-translational modification of specific regulatory proteins. The exact mechanism of this signaling is unclear, but increased understanding offers the potential to promote or inhibit apoptosis through modulating the redox environment of cells. Peroxiredoxins are thiol peroxidases that remove hydroperoxides, and are also emerging as important players in cellular redox signaling. This review discusses the potential role of peroxiredoxins in the regulation of apoptosis, and also their ability to act as biomarkers of redox changes during the initiation and progression of cell death.
\end{abstract}

\section{INTRODUCTION}

Cells employ a complex array of antioxidants to protect themselves from oxidative stress. If these defenses become overwhelmed then damage and cell death ensue. High levels of oxidative stress quickly compromise major antioxidant systems and necrosis occurs. At lower levels cells are able to sense oxidative damage and activate inbuilt death pathways, enabling orderly disposal of the affected cell. Apoptosis is the most extensively studied of the regulated cell death programmes. It is important for the clearance of damaged and infected cells, and also for maintaining tissue homeostasis by removing unwanted cells. Interestingly, intracellular redox changes are proposed to be a significant event during apoptosis signaling, regardless of the initiating stimulus. In this scenario reactive oxygen species act as second messengers, with a carefully regulated increase in generation mediating the post-translational modification of specific regulatory proteins. The exact mechanism of this signaling is unclear, but increased understanding offers the possibility of promoting or inhibiting apoptosis through modulating

Centre for Free Radical Research, Department of Pathology, University of Otago, Christchurch 8140, New Zealand

*Correspondence: mark.hampton@otago.ac.nz

Received 21 December, 2015; accepted 24 December, 2015; published online 25 January, 2016

Keywords: apoptosis, hydrogen peroxide, mitochondria, peroxiredoxins, redox signaling the redox environment of cells.

Redox signaling is implicated in a number of biological processes, including growth factor signaling, cell differentiation and cytokine production (Finkel, 2000). Hydrogen peroxide is widely recognized as a signaling species, with cysteine residues the important targets. Cysteine oxidation leads to the structural modification and altered activity of a variety of proteins, including transcription factors, kinases, phosphatases, metabolic enzymes, membrane channels and cytoskeletal proteins. Cysteine reactivity is strongly influenced by the local protein microenvironment, and kinetic hierarchies enabling selective modification of proteins. However, it is apparent that many of the proteins oxidized by hydrogen peroxide are several orders of magnitude less reactive than the abundant cellular peroxidases that remove hydrogen peroxide (Cox et al., 2010; Winterbourn, 2008; Winterbourn and Hampton, 2008). This suggests more complex mechanisms for selective oxidation. Of particular interest is the peroxiredoxins (Prxs); abundant thiol peroxidases that react with hydrogen peroxide $10^{6}-10^{7}$ times faster than free cysteine (Cox et al., 2009; Ogusucu et al., 2007; Parsonage et al., 2005; Peskin et al., 2007). Prxs reduce hydroperoxides to water, and are important players in antioxidant defence (Rhee et al., 2005; Wood et al., 2003b). However, the Prxs have additional features that make them potential signaling proteins. This review discusses the potential role of Prxs in apoptosis signaling, and also their ability to act as biomarkers of redox changes during the initiation and progression of cell death.

\section{REDOX SIGNALING AND APOPTOSIS}

The ability of a cell to undergo apoptosis is tightly regulated, with the "decision" influenced by a range of environmental and internal inputs. Apoptosis signaling pathways converge on activation of the caspases, a family of cysteine-dependent aspartate proteases (Salvesen and Dixit, 1997). Effector caspases such as caspase- 3 play a critical role in apoptosis by cleaving key structural and regulatory proteins. They are kept as inactive pro-caspases in healthy cells, and only become activated following proteolytic cleavage by initiator caspases. The initiator caspases themselves become activated upon recruitment to death receptor-associated signaling complexes (caspase-8), or apoptosomes that form upon the release of cytochrome $c$ from mitochondria (caspase-9). The release of cytochrome $c$ and other pro-apoptotic factors is mediated by Bax and Bak, members of the Bcl-2 protein family that create pores in the mitochondrial outer membrane. Pore formation is controlled by the proand anti-apoptotic members of the $\mathrm{Bcl}-2$ family that initiates 
or impairs pore formation respectively (Adams and Cory, 1998). Apoptotic stimuli increase the levels or activity of pro-apoptotic proteins such as Bim or Bid, while increased expression of antiapoptotic proteins such as Bcl-2 increase the resistance of cells.

Reactive oxygen species (ROS) can directly trigger apoptosis by causing excessive protein, lipid and nucleic acid oxidation, but just as importantly, they have the potential to regulate the pathways utilized by other apoptotic stimuli. Cysteine oxidation alters the catalytic activity of various enzymes, and manipulates the structure of other proteins. Targets within the core components of the death machinery include $\mathrm{Bcl}-2$ family members (D'Alessio et al., 2005) and caspases (Hampton and Orrenius, 1997; Hampton et al., 2002). Other relevant redox-sensitive targets include tyrosine phosphatases that regulate kinase signaling cascades (Meng et al., 2002), and transcription factors that influence the expression of pro- and anti-apoptotic proteins (Scotcher et al., 2011). Oxidation of the anionic phospholipid cardiolipin in the inner mitochondrial membrane is also proposed to play an important role in mitochondrial disruption and cytochrome $\mathrm{c}$ release that occurs during apoptosis (Kagan et al., 2005).

Evidence for a role of redox signaling in apoptosis requires (1) early disruption of redox homeostasis resulting in an oxidation event that promotes apoptosis, and (2) specific prevention of the oxidation event resulting in inhibition of apoptosis. Increased ROS generation has been reported during apoptosis, but the assays are complicated and can be difficult to accurately interpret. For example, 2',7'-dichlorofluorescein (DCF) oxidation is commonly used to measure increased levels of hydrogen peroxide. However, the reaction is not direct, and is instead catalyzed by peroxidases or transition metals and involves intermediate radicals that can be scavenged within the cell (Bonini et al., 2006). Changes in any of these factors, for example the release of the cytochrome $c$ from mitochondria, with its inherent peroxidase activity, can increase DCF oxidation without altering hydrogen peroxide levels within a cell (Burkitt and Wardman, 2001).

It can also be difficult to distinguish if increased oxidation is an early event in apoptosis signaling or a consequence of cell death. Any process that compromises the antioxidant systems of cells, for example a loss of NADPH or glutathione, or leads to increased ROS generation from damaged mitochondria or lysosomes, will disrupt redox homeostasis. This is not to undermine the significance of redox changes that occur as a consequence of apoptosis. They can serve as markers of apoptosis initiation, and they may also assist with amplifying apoptosis signaling. For example, it has been reported that oxidants from individual mitochondria releasing pro-apoptotic factors sensitize adjacent mitochondria to Bid during receptor-mediated apoptosis (Garcia-Perez et al., 2012).

Testing the significance of redox changes requires inhibition of oxidation and assessment of the subsequent effects on apoptosis. Compounds with antioxidant properties have been reported to inhibit apoptosis induced by non-oxidative triggers, but selectivity is a major limitation of this approach. The term "antioxidant" describes a broad range of compounds that block the biological activity of different ROS through inhibiting generation, scavenging or repairing damaged constituents. Their mechanism of action can be diverse, and results in non-specific effects. For example, $\mathrm{N}$-acetylcysteine (NAC) is reported to inhibit apoptosis in different models, but it is unclear how it is acting. The commonly described but least likely mechanism is direct scavenging of hydrogen peroxide. It is possible that the millimolar levels of NAC required to inhibit apoptosis influene the redox status of structural cysteines in extracellular receptors
(Hayakawa et al., 2003). The most convincing evidence would be selective impairment of a defined oxidation event shown to occur during apoptosis, but more knowledge of the molecular mechanisms of redox signaling pathways is necessary before this can be attempted.

\section{PEROXIREDOXINS: ANTIOXIDANTS AND REDOX SENSORS}

Prxs are a ubiquitous family of antioxidant proteins that use specialized cysteine residues to breakdown hydroperoxides (Rhee et al., 2005; Wood et al., 2003b). Mammals have six Prxs and transgenic mouse models show that there is little redundancy in the family, with the loss of individual Prxs leading to haematological disorders and cancer, and also increasing susceptibility to diseases associated with oxidative stress (Lee et al., 2003; Li et al., 2007; Neumann et al., 2003). The peroxidatic cysteine in the active site of Prxs is extremely sensitive to oxidation, forming a sulfenic acid upon exposure to hydrogen peroxide. A disulfide bond is then formed with the resolving cysteine of an adjacent Prx, resulting in an intermolecular dimer The catalytic cycle is completed by reduction of the dimer by thioredoxin. Prxs are highly abundant proteins, and as such they are considered to be an important component of cellular antioxidant networks.

Interestingly, however, mammalian Prxs have evolved to be easily inactivated by hydrogen peroxide (Wood et al., 2003a). In comparison to prokaryotic Prxs, structural constraints slow disulfide bond formation making the sulfenic acid susceptible to sulfinylation by a second molecule of hydrogen peroxide. Hyperoxidized Prxs remain catalytically inactive until slowly reduced by sulfiredoxin. This begs the question of why an antioxidant protein has evolved to be inactivated by a substrate that it can effectively remove. Furthermore, Prxs form intricate quaternary structures including doughnut-shaped decameric or dodecameric complexes that can stack (Radjainia et al., 2015). These structures are disrupted upon oxidation or phosphorylation (Wood et al., 2002). Hyperoxidation stabilizes the complexes and also imparts chaperone activity (Jang et al., 2004). All of this seems unduly complex for a peroxide-removal system, and has led to speculation that Prxs are hydrogen peroxide sensors that play a central role in redox signaling.

Several mechanisms have been proposed for Prx involvement in redox signaling. Wood et al. proposed the floodgate model (Wood et al., 2003a), wherein Prxs consume the low endogenous levels of hydrogen peroxide in cells, but become inactivated as levels rise, leading to an upsurge in the hydrogen peroxide available for oxidizing less reactive thiol proteins Alternatively, Prx structural changes following oxidation could lead to altered protein-protein interactions and the propagation of a signal. There is growing interest in Prxs contributing to redox relays, in which these abundant proteins are the primary target of hydrogen peroxide, and then undergo disulfide exchange reactions leading to the oxidation of specific interacting proteins. Various examples of this mechanism have been reported (Delaunay et al., 2002; Sobotta et al., 2015), including the ability of Prx1 to facilitate oxidation of the apoptosis signaling kinase (ASK1) (Jarvis et al., 2012). To date there is no evidence for any of these pathways playing a significant role in apoptosis.

\section{PEROXIREDOXINS AND APOPTOSIS}

Knockdown of Prx expression typically makes cells more susceptible to cell death induced by oxidative stress, while Prx 
overexpression provides protection. This is consistent with the antioxidant properties of these proteins. Interestingly, however, early studies showed that Prx overexpression also delayed apoptosis in cells deprived of serum or growth factors (Ichimiya et al., 1997; Zhang et al., 1997), which did not cause an oxidative stress. Several other studies have shown similar effects with a broad range of cytotoxic agents. These observations support a mechanism whereby endogenous hydroperoxides are generated during the initiation of apoptosis and their removal significantly impedes the process.

Mitochondria are considered to be a significant source of hydrogen peroxide within cells. Prx 3 is a mitochondrial member of the Prx family, and is one of the major targets of hydrogen peroxide generated within the mitochondrial matrix (Cox et al., 2010). Overexpression of Prx3 was shown to provide protection against induction of apoptosis by serum deprivation, ceramide or etoposide (Zhang et al., 1997), and a related study showed protection against hypoxia and redox-active compounds (Nonn et al., 2003). The abrin protein was hypothesized to trigger apoptosis by binding and inactivating Prx3 (AOP-1) (Shih et al., 2001), and Prx3 has previously been identified as a target gene of the oncogenic transcription factor c-Myc essential for Mycmediated transformation (Wonsey et al., 2002). Prx 3 depletion by siRNA led to a more rapid release of cytochrome $c$ upon addition of staurosporine or TNF (Chang et al., 2004). The antiapoptotic effect of Prx 3 is dependent on catalytic cycling, as overexpression of a dominant negative thioredoxin 2 (C93S), which forms a catalytically inactive disulfide bond with $\operatorname{Prx} 3$, sensitizes cells to tert-butyl hydroperoxide and TNF-induced apoptosis (Zhang et al., 2007).

Increased Prx3 expression is reported in breast and lung carcinomas (Noh et al., 2001; Park et al., 2006), prostate (Whitaker et al., 2013), ovarian (Wang et al., 2013), cervical (Hu et al., 2013), endometrial (Han et al., 2012), mesothelioma (Kinnula et al., 2002) and hepatocellular carcinomas (Choi et al., 2002). Also, the developmental abnormalities and malignancies associated with Fanconi anemia in humans has also been closely linked to deregulation of Prx3 function (Mukhopadhyay et al., 2006). This raises the possibility that inhibiting Prxs, particularly Prx3, may provide an alternate strategy for targeting cancer cells that are resistant to undergoing cell death. Early reports indicate promising results in pre-clinical studies with compounds that can target Prxs (Cunniff et al., 2015; Li et al., 2015; Liu et al., 2012; Trzeciecka et al., 2015). Compounds that promote Prx oxidation through compromising thiol reduction pathways (Brown et al., 2008; Cox et al., 2008a; Myers, 2015), may also have useful applications in promoting cell death.

In addition to regulating apoptosis sensitivity, the Prxs are useful markers of altered redox homeostasis. The disulfidelinked dimers that form upon Prx oxidation can be visualized by western blotting of non-reducing SDS-PAGE gels, and have been proved useful for monitoring the redox state of cellular Prxs (Poynton and Hampton, 2014). We observed the accumulation of oxidized Prx 3 during Fas-mediated apoptosis in Jurkat T-lymphoma cells (Cox et al., 2008b). The timing of Prx 3 oxidation corresponded to cytochrome $c$ release and caspase activation, but came well before the global oxidative stress associated with end-stage apoptosis. The specific compartmentalisation of peroxiredoxin family members can also provide information on the intracellular sites of redox changes. While mitochondrial $\operatorname{Prx} 3$ accumulated in its oxidised form, there was no oxidation of the cytoplasmic Prxs, suggesting specific disruption of mitochondrial redox homeostasis in this model (Cox et al., 2008b).

\section{CONCLUSION AND FUTURE DIRECTIONS}

A significant amount of published data supports a model in which steady state levels of hydrogen peroxide increase during the initiation of apoptosis, and this acts as a signaling molecule to promote cell death. Unfortunately, much of this data is indirect, and relies on non-specific inhibitors and probes. It is also difficult to separate causative processes from those that occur as a consequence of cell death. To determine the importance of intracellular redox changes in apoptosis, it is crucial that the precise mechanisms are unraveled, thereby enabling the development of selective inhibitors. The Prxs appear to play a significant role in redox signaling, and the disruption of their catalytic activity and protein interactions may prove to be a valuable tool.

While this review was focused on apoptosis, recent years have seen an increased recognition that a variety of regulated cell death pathways exist (Vanden Berghe et al., 2014). The mechanistic details of these pathways are only beginning to be elucidated, but redox-dependent events are implicated in several of them. The best studied to date is necroptosis (Degterev et al., 2005), the cell death pathway characterized by activation of the mixed lineage kinase domain-like (MLKL) pseudokinase (Sun et al., 2012). Protein receptor interacting protein kinases (RIPKs) phosphorylate MLKL, promoting its oligomerization and membrane association, followed by a loss of membrane integrity (Murphy et al., 2013; Wang et al., 2014). Necroptosis can be triggered through death receptors such as TNF, in the presence of caspase inhibitors. Early studies of TNF-mediated necroptosis reported elevated ROS levels, but the source, timing and role in execution of cell death remains controversial. Mitochondrial ROS have been implicated (Goossens et al., 1995; Schulze-Osthoff et al., 1992), as have oxidants derived from NOX2 (Kim et al., 2007), and the radical scavenger butylated hydroxyanisole can inhibit TNF-induced necroptosis in some cell types (Lin et al., 2004). Studies on the role of Prxs in necroptosis are warranted, as is their ability to act as markers of redox disruption prior to the induction of cell death.

\section{ACKNOWLEDGMENTS}

The authors' investigations on the role of peroxiredoxins in cell death are currently supported by the Marsden Fund Council from Government funding, administered by the Royal Society of New Zealand.

\section{REFERENCES}

Adams, J.M., and Cory, S. (1998). The Bcl-2 protein family: arbiters of cell survival. Science 281, 1322-1326

Bonini, M.G., Rota, C., Tomasi, A., and Mason, R.P. (2006). The oxidation of 2',7'-dichlorofluorescin to reactive oxygen species: a self-fulfilling prophesy? Free Radic. Biol. Med. 40, 968-975.

Brown, K.K., Eriksson, S.E., Arner, E.S., and Hampton, M.B. (2008) Mitochondrial peroxiredoxin 3 is rapidly oxidized in cells treated with isothiocyanates. Free Radic. Biol. Med. 45, 494-502.

Burkitt, M.J., and Wardman, P. (2001). Cytochrome C is a potent catalyst of dichlorofluorescin oxidation: implications for the role of reactive oxygen species in apoptosis. Biochem. Biophys. Res. Commun. 282, 329-333.

Chang, T.S., Cho, C.S., Park, S., Yu, S.Q., Kang, S.W., and Rhee, S.G. (2004). Peroxiredoxin III, a mitochondrion-specific peroxidase, regulates apoptotic signaling by mitochondria. J. Biol. Chem. 279, 41975-41984

Choi, J.H., Kim, T.N., Kim, S., Baek, S.H., Kim, J.H., Lee, S.R., and Kim, J.R. (2002). Overexpression of mitochondrial thioredoxin reductase and peroxiredoxin III in hepatocellular carcinomas. Anticancer Res. 22, 3331-3335. 
Cox, A.G., Brown, K.K., Arner, E.S., and Hampton, M.B. (2008a). The thioredoxin reductase inhibitor auranofin triggers apoptosis through a Bax/Bak-dependent process that involves peroxiredoxin 3 oxidation. Biochem. Pharmacol. 76, 1097-1109.

Cox, A.G., Pullar, J.M., Hughes, G., Ledgerwood, E.C., and Hampton, M.B. (2008b). Oxidation of mitochondrial peroxiredoxin 3 during the initiation of receptor-mediated apoptosis. Free Radic. Biol. Med. 44, 1001-1009.

Cox, A.G., Peskin, A.V., Paton, L.N., Winterbourn, C.C., and Hampton, M.B. (2009). Redox potential and peroxide reactivity of human peroxiredoxin 3. Biochemistry 48, 6495-6501.

Cox, A.G., Winterbourn, C.C., and Hampton, M.B. (2010). Mitochondrial peroxiredoxin involvement in antioxidant defence and redox signalling. Biochem. J. 425, 313-325.

Cunniff, B., Newick, K., Nelson, K.J., Wozniak, A.N., Beuschel, S., Leavitt, B., Bhave, A., Butnor, K., Koenig, A., Chouchani, E.T., et al. (2015). Disabling Mitochondrial Peroxide Metabolism via Combinatorial Targeting of Peroxiredoxin 3 as an Effective Therapeutic Approach for Malignant Mesothelioma. PLoS One 10, e0127310.

D'Alessio, M., De Nicola, M., Coppola, S., Gualandi, G., Pugliese, L., Cerella, C., Cristofanon, S., Civitareale, P., Ciriolo, M.R., Bergamaschi, A., et al. (2005). Oxidative Bax dimerization promotes its translocation to mitochondria independently of apoptosis. FASEB J. 19, 1504-1506.

Degterev, A., Huang, Z., Boyce, M., Li, Y., Jagtap, P., Mizushima, N., Cuny, G.D., Mitchison, T.J., Moskowitz, M.A., and Yuan, J. (2005). Chemical inhibitor of nonapoptotic cell death with therapeutic potential for ischemic brain injury. Nat. Chem. Biol. 1, 112119

Delaunay, A., Pflieger, D., Barrault, M.B., Vinh, J., and Toledano, M.B. (2002). A thiol peroxidase is an $\mathrm{H} 2 \mathrm{O} 2$ receptor and redoxtransducer in gene activation. Cell 111, 471-481.

Finkel, T. (2000). Redox-dependent signal transduction. FEBS Lett. $476,52-54$

Garcia-Perez, C., Roy, S.S., Naghdi, S., Lin, X., Davies, E. and Hajnoczky, G. (2012). Bid-induced mitochondrial membrane permeabilization waves propagated by local reactive oxygen species (ROS) signaling. Proc. Natl. Acad. Sci. USA 109, 44974502.

Goossens, V., Grooten, J., De Vos, K., and Fiers, W. (1995). Direct evidence for tumor necrosis factor-induced mitochondrial reactive oxygen intermediates and their involvement in cytotoxicity. Proc. Natl. Acad. Sci. USA 92, 8115-8119.

Hampton, M.B., and Orrenius, S. (1997). Dual regulation of caspase activity by hydrogen peroxide: implications for apoptosis. FEBS Lett. 414, 552-556.

Hampton, M.B., Stamenkovic, I., and Winterbourn, C.C. (2002). Interaction with substrate sensitises caspase-3 to inactivation by hydrogen peroxide. FEBS Lett. 517, 229-232.

Han, S., Shen, H., Jung, M., Hahn, B.S., Jin, B.K., Kang, I., Ha, J., and Choe, W. (2012). Expression and prognostic significance of human peroxiredoxin isoforms in endometrial cancer. Oncol. Lett. 3, 1275-1279.

Hayakawa, M., Miyashita, H., Sakamoto, I., Kitagawa, M., Tanaka, H., Yasuda, H., Karin, M., and Kikugawa, K. (2003). Evidence that reactive oxygen species do not mediate NF-kappaB activation. EMBO J. 22, 3356-3366.

$\mathrm{Hu}$, J.X., Gao, Q., and Li, L. (2013). Peroxiredoxin 3 is a novel marker for cell proliferation in cervical cancer. Biomed. Rep. 1, 228-230.

Ichimiya, S., Davis, J.G., O'Rourke, D.M., Katsumata, M., and Greene, M.I. (1997). Murine thioredoxin peroxidase delays neuronal apoptosis and is expressed in areas of the brain most susceptible to hypoxic and ischemic injury. DNA Cell Biol. 16, 311321

Jang, H.H., Lee, K.O., Chi, Y.H., Jung, B.G., Park, S.K., Park, J.H., Lee, J.R., Lee, S.S., Moon, J.C., Yun, J.W., et al. (2004). Two enzymes in one; two yeast peroxiredoxins display oxidative stress-dependent switching from a peroxidase to a molecular chaperone function. Cell 117, 625-635.

Jarvis, R.M., Hughes, S.M., and Ledgerwood, E.C. (2012). Peroxiredoxin 1 functions as a signal peroxidase to receive, transduce, and transmit peroxide signals in mammalian cells. Free Radic. Biol. Med. 53, 1522-1530.

Kagan, V.E., Tyurin, V.A., Jiang, J., Tyurina, Y.Y., Ritov, V.B., Amos- cato, A.A., Osipov, A.N., Belikova, N.A., Kapralov, A.A., Kini, V., et al. (2005). Cytochrome $c$ acts as a cardiolipin oxygenase required for release of proapoptotic factors. Nat. Chem. Biol. 1 223-232.

Kim, Y.S., Morgan, M.J., Choksi, S., and Liu, Z.G. (2007). TNFinduced activation of the Nox1 NADPH oxidase and its role in the induction of necrotic cell death. Mol. Cell 26, 675-687.

Kinnula, V.L., Lehtonen, S., Sormunen, R., Kaarteenaho-Wiik, R. Kang, S.W., Rhee, S.G., and Soini, Y. (2002). Overexpression of peroxiredoxins I, II, III, V, and VI in malignant mesothelioma. J. Pathol. 196, 316-323.

Lee, T.H., Kim, S.U., Yu, S.L., Kim, S.H., Park, D.S., Moon, H.B., Dho, S.H., Kwon, K.S., Kwon, H.J., Han, Y.H., et al. (2003). Peroxiredoxin II is essential for sustaining life span of erythrocytes in mice. Blood 101, 5033-5038.

Li, L., Shoji, W., Takano, H., Nishimura, N., Aoki, Y., Takahashi, R. Goto, S., Kaifu, T., Takai, T., and Obinata, M. (2007). Increased susceptibility of MER5 (peroxiredoxin III) knockout mice to LPSinduced oxidative stress. Biochem. Biophys. Res. Commun. 355, 715-721.

Li, G., Xie, B., Li, X., Chen, Y., Xu, Y., Xu-Welliver, M., and Zou, L. (2015). Downregulation of peroxiredoxin-1 by beta-elemene enhances the radiosensitivity of lung adenocarcinoma xenografts. Oncol. Rep. 33, 1427-1433.

Lin, Y., Choksi, S., Shen, H.M., Yang, Q.F., Hur, G.M., Kim, Y.S. Tran, J.H., Nedospasov, S.A., and Liu, Z.G. (2004). Tumor necrosis factor-induced nonapoptotic cell death requires receptorinteracting protein-mediated cellular reactive oxygen species accumulation. J. Biol. Chem. 279, 10822-10828.

Liu, C.X., Yin, Q.Q., Zhou, H.C., Wu, Y.L., Pu, J.X., Xia, L., Liu, W. Huang, X., Jiang, T., Wu, M.X., et al. (2012.) Adenanthin targets peroxiredoxin I and II to induce differentiation of leukemic cells Nat. Chem. Biol. 8, 486-493.

Meng, T.C., Fukada, T., and Tonks, N.K. (2002). Reversible oxidation and inactivation of protein tyrosine phosphatases in vivo Mol. Cell 9, 387-399.

Mukhopadhyay, S.S., Leung, K.S., Hicks, M.J., Hastings, P.J., Youssoufian, H., and Plon, S.E. (2006). Defective mitochondria peroxiredoxin-3 results in sensitivity to oxidative stress in Fanconi anemia. J. Cell Biol. 175, 225-235.

Murphy, J.M., Czabotar, P.E., Hildebrand, J.M., Lucet, I.S., Zhang J.G., Alvarez-Diaz, S., Lewis, R., Lalaoui, N., Metcalf, D., Webb, A.I., et al. (2013). The pseudokinase MLKL mediates necroptosis via a molecular switch mechanism. Immunity 39, 443-453.

Myers, C.R. (2015). Enhanced targeting of mitochondrial peroxide defense by the combined use of thiosemicarbazones and inhibitors of thioredoxin reductase. Free Radic. Biol. Med. 91, 81-92.

Neumann, C.A., Krause, D.S., Carman, C.V., Das, S., Dubey, D.P., Abraham, J.L., Bronson, R.T., Fujiwara, Y., Orkin, S.H., and Van Etten, R.A. (2003). Essential role for the peroxiredoxin Prdx1 in erythrocyte antioxidant defence and tumour suppression. Nature 424, 561-565.

Noh, D.Y., Ahn, S.J., Lee, R.A., Kim, S.W., Park, I.A., and Chae, H.Z. (2001). Overexpression of peroxiredoxin in human breas cancer. Anticancer Res. 21, 2085-2090.

Nonn, L., Berggren, M., and Powis, G. (2003). Increased expression of mitochondrial peroxiredoxin-3 (thioredoxin-peroxidase-2) protects cancer cells against hypoxia and drug-induced hydrogen peroxide-dependent apoptosis. Mol. Cancer Res. 1, 682-689.

Ogusucu, R., Rettori, D. Munhoz, D.C., Netto, L.E, and Augusto, O. (2007). Reactions of yeast thioredoxin peroxidases I and II with hydrogen peroxide and peroxynitrite: rate constants by competitive kinetics. Free Radic. Biol. Med. 42, 326-334.

Park, J.H., Kim, Y.S., Lee, H.L., Shim, J.Y., Lee, K.S., Oh, Y.J., Shin, S.S., Choi, Y.H., Park, K.J., Park, R.W., et al. (2006). Expression of peroxiredoxin and thioredoxin in human lung cancer and paired normal lung. Respirology 11, 269-275.

Parsonage, D., Youngblood, D.S., Sarma, G.N., Wood, Z.A., Karplus, P.A., and Poole, L.B. (2005). Analysis of the link between enzymatic activity and oligomeric state in AhpC, a bacterial peroxiredoxin. Biochemistry 44, 10583-10592.

Peskin, A.V., Low, F.M., Paton, L.N., Maghzal, G.J., Hampton, M.B., and Winterbourn, C.C. (2007). The high reactivity of peroxiredoxin 2 with $\mathrm{H}(2) \mathrm{O}(2)$ is not reflected in its reaction with other oxidants and thiol reagents. J. Biol. Chem. 282, 11885-11892.

Poynton, R.A., and Hampton, M.B. (2014). Peroxiredoxins as bio- 
markers of oxidative stress. Biochim. Biophys. Acta 1840, 906912.

Radjainia, M., Venugopal, H., Desfosses, A., Phillips, A.J., Yewdall, N.A., Hampton, M.B., Gerrard, J.A., and Mitra, A.K. (2015) Cryo-electron microscopy structure of human peroxiredoxin-3 filament reveals the assembly of a putative chaperone. Structure 23, 912-920

Rhee, S.G., Chae, H.Z., and Kim, K. (2005). Peroxiredoxins: a historical overview and speculative preview of novel mechanisms and emerging concepts in cell signaling. Free Radic. Biol. Med. 38, 1543-1552.

Salvesen, G.S., and Dixit, V.M. (1997). Caspases: intracellular signaling by proteolysis. Cell $91,443-446$.

Schulze-Osthoff, K., Bakker, A.C. Vanhaesebroeck, B., Beyaert, R. Jacob, W.A., and Fiers, W. (1992). Cytotoxic activity of tumor necrosis factor is mediated by early damage of mitochondrial functions. Evidence for the involvement of mitochondrial radical generation. J. Biol. Chem. 267, 5317-5323.

Scotcher, J., Clarke, D.J., Weidt, S.K., Mackay, C.L., Hupp, T.R., Sadler, P.J., and Langridge-Smith, P.R. (2011). Identification of two reactive cysteine residues in the tumor suppressor protein p53 using top-down FTICR mass spectrometry. J. Am. Soc. Mass Spectrom. 22, 888-897.

Shih, S.F., Wu, Y.H., Hung, C.H., Yang, H.Y., and Lin, J.Y. (2001). Abrin triggers cell death by inactivating a thiol-specific antioxidant protein. J. Biol. Chem. 276, 21870-21877.

Sobotta, M.C., Liou, W., Stocker, S., Talwar, D., Oehler, M., Ruppert, T., Scharf, A.N., and Dick, T.P. (2015). Peroxiredoxin-2 and STAT3 form a redox relay for $\mathrm{H} 2 \mathrm{O} 2$ signaling. Nat. Chem. Biol. 11, 64-70.

Sun, L., Wang, H., Wang, Z., He, S., Chen, S., Liao, D., Wang, L., Yan, J., Liu, W., Lei, X., et al. (2012). Mixed lineage kinase domain-like protein mediates necrosis signaling downstream of RIP3 kinase. Cell 148, 213-227.

Trzeciecka, A., Klossowski, S., Bajor, M., Zagozdzon, R., Gaj, P., Muchowicz, A., Malinowska, A., Czerwoniec, A., Barankiewicz, J., Domagala, A., et al. (2015). Dimeric peroxiredoxins are druggable targets in human Burkitt lymphoma. Oncotarget [Epub ahead of print]

Vanden Berghe, T., Linkermann, A., Jouan-Lanhouet, S., Walczak,
$\mathrm{H}$., and Vandenabeele, P. (2014). Regulated necrosis: the expanding network of non-apoptotic cell death pathways. Nat.Rev. Mol. Cell Biol. 15, 135-147.

Wang, X.Y., Wang, H.J., and Li, X.Q. (2013). Peroxiredoxin III protein expression is associated with platinum resistance in epithelial ovarian cancer. Tumour Biol. 34, 2275-2281.

Wang, H., Sun, L., Su, L., Rizo, J., Liu, L., Wang, L.F., Wang, F.S., and Wang, X. (2014). Mixed lineage kinase domain-like protein MLKL causes necrotic membrane disruption upon phosphorylation by RIP3. Mol. Cell 54, 133-146.

Whitaker, H.C., Patel, D., Howat, W.J., Warren, A.Y., Kay, J.D. Sangan, T., Marioni, J.C., Mitchell, J., Aldridge, S., Luxton, H.J., et al. (2013). Peroxiredoxin-3 is overexpressed in prostate cancer and promotes cancer cell survival by protecting cells from oxidative stress. Br. J. Cancer 109, 983-993.

Winterbourn, C.C. (2008). Reconciling the chemistry and biology of reactive oxygen species. Nat. Chem. Biol. 4, 278-286.

Winterbourn, C.C., and Hampton, M.B. (2008). Thiol chemistry and specificity in redox signaling. Free Radic. Biol. Med. 45, 549-561.

Wonsey, D.R., Zeller, K.I., and Dang, C.V. (2002). The c-Myc target gene PRDX3 is required for mitochondrial homeostasis and neoplastic transformation. Proc. Natl. Acad. Sci. USA 99, 66496654

Wood, Z.A., Poole, L.B., Hantgan, R.R. and Karplus, P.A. (2002). Dimers to doughnuts: redox-sensitive oligomerization of 2cysteine peroxiredoxins. Biochemistry 41, 5493-5504.

Wood, Z.A., Poole, L.B., and Karplus, P.A. (2003a). Peroxiredoxin evolution and the regulation of hydrogen peroxide signaling. Science 300, 650-653.

Wood, Z.A., Schroder, E., Harris, J.R., and Poole, L.B. (2003b) Structure, mechanism and regulation of peroxiredoxins. Trends Biochem. Sci. 28, 32-40.

Zhang, P., Liu, B., Kang, S.W., Seo, M.S., Rhee, S.G., and Obeid L.M. (1997). Thioredoxin peroxidase is a novel inhibitor of apoptosis with a mechanism distinct from that of Bcl-2. J. Biol. Chem. 272, 30615-30618.

Zhang, H., Go, Y.M., and Jones, D.P. (2007). Mitochondrial thioredoxin-2/peroxiredoxin-3 system functions in parallel with mitochondrial GSH system in protection against oxidative stress. Arch. Biochem. Biophys. 465, 119-126. 